\title{
Current management of Duchenne muscular dystrophy in the Middle East: expert report
}

Mohammed Al Jumah¹, Mohammad Al Muhaizea², Ahmed Al Rumayyan³, Abdulaziz Al

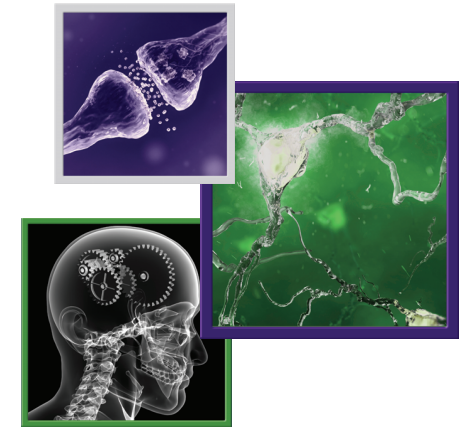
Saman ${ }^{1}$, Ali Al Shehri², Edward Cupler*,4, Mohammed Jan ${ }^{5}$, Abubaker Al Madani ${ }^{6}$, Waseem Fathalla ${ }^{7}$, Pawan Kashyape ${ }^{8}$, Gururaj Kodavooru ${ }^{8}$, Khalid Al Thihli ${ }^{9}$, Laila Bastaki $^{10}$, Andre Megarbane ${ }^{11}$, Cristina Skrypnyk ${ }^{12}$, Gholamreza Zamani ${ }^{13}$, Sylvie Tuffery-Giraud $^{14}$, Andoni Urtizberea ${ }^{15}$ \& Carlos Ignacio Ortez González ${ }^{16}$

${ }^{1}$ King Fahad Medical City, Riyadh, Saudi Arabia

${ }^{2}$ King Faisal Specialist Hospital \& Research Centre, Riyadh, Saudi Arabia

${ }^{3}$ National Guard Health Affairs, Riyadh, Saudi Arabia

${ }^{4}$ King Faisal Specialist Hospital \& Research Centre, Tihamah, Saudi Arabia

${ }^{5}$ Pedia Care Clinic, Tihamah, Saudi Arabia

${ }^{6}$ Rashid Hospital, Dubai, United Arab Emirates

${ }^{7}$ Mafraq Hospital, Abu Dhabi, United Arab Emirates

${ }^{8}$ Latifa Hospital, Dubai, United Arab Emirates

${ }^{9}$ Sultan Qaboos University Hospital, Genetic Centre, Muscat, Oman

${ }^{10}$ Kuwait Medical Genetic Centre, Kuwait

${ }^{11}$ Université Saint-Joseph, Beirut, Lebanon

${ }^{12}$ Center of Princess Al-Jawhara (ACMID), Arabian Gulf University, Bahrain

${ }^{13}$ Children's Medical Center, Tehran University of Medical Sciences, Tehran, Iran

${ }^{14}$ Laboratoire de Génétique de Maladies Rares IURC, Occitanie, France

${ }^{15}$ Hôpital Marin de Hendaye, Route de la Corniche, 64700 Hendaye, France

${ }^{16}$ Unidad de Patología Neuromuscular Servicio de Neurología, Hospital Sant Joan de Déu, Catalonia, Spain

*Author for correspondence: Tel.: +966 550793 034; cuplere@gmail.com

\begin{abstract}
Aim: Duchenne muscular dystrophy (DMD) is a severe and rare X-linked neuromuscular childhood disorder that results in functional decline, loss of ambulation and early death due to cardiac or respiratory failure. The objective of this paper is to address different aspects of the current management of DMD in the Middle East, north Africa (MENA) region, and to gather experts' recommendations on how to optimally diagnose and treat patients suffering from this disease. Methods: A group of experts (neuromuscular medicine, neuropediatricians and geneticists) convened to discuss the diagnosis and management of DMD in the MENA region. A list of practical statements was prepared by the chair of the meeting to guide the discussions around critical aspects relating to the current and future management of DMD. Results \& conclusion: Ideally, DMD management should be a multidisciplinary approach. Nevertheless, few tertiary care hospitals in the region are currently able to provide the full spectrum of medical expertise and services needed by DMD patients. Clinical practice in the region remains heterogeneous. Specific guidelines for diagnosis and treatment are needed in the MENA region to improve outcomes. Disease awareness among the general public and the medical community is lacking. Now that mutation-specific therapies are being developed and more widely studied, general education programs regarding early signs and symptoms, a standardized referral and diagnosis pathway, patient registries and support groups will significantly improve the management of the disease.
\end{abstract}

First draft submitted: 17 January 2019; Accepted for publication: 2 April 2019; Published online: 5 June 2019

Keywords: Duchenne muscular dystrophy $\bullet$ dystrophy $\bullet$ genetic disorders $\bullet$ multidisciplinary care $\bullet$ muscle degeneration • muscle weakness • treatment guidelines

Duchenne muscular dystrophy (DMD) is a clinically heterogeneous, severe and rare X-linked recessive neuromuscular childhood disorder that occurs in 1 in every 3600-6000 live male births [1]. The disease results in functional decline, most notably progressive muscle weakness, and is associated with behavioral and learning disabilities in 
approximately a third of cases [2]. A link between mutation location within the DMD gene (e.g., exon 52) and cognitive deficit was noted in the 1990s. Subsequent studies have shown that rather than a specific deletion involving exon 52, deletions localized in the second half of the gene were more frequently associated with lower IQ than those in the first half of the gene, but no specific genotype/phenotype relationship was identified [3]. Most patients become wheelchair-bound and dependent on others for their daily activities during the second decade of life, and usually die prematurely as a result of cardiac or respiratory failure [4,5].

This paper is based on a DMD Experts' Meeting held in Dubai in September 2016. The goals of the meeting were twofold:

- First, to ascertain the current status of DMD patient management in Middle East, North Africa (MENA) countries, and the region overall, based on feedback from the expert group;

- Second, to make recommendations to develop initiatives that can be implemented in the MENA region to support physicians in their quest to improve the standards of care for individuals with DMD.

The participants were experts in this clinical setting and were either specialists directly involved in the regular care of patients with DMD in the MENA region or consultants from other regions with vast experience managing patients with DMD (Supplementary Materials 1). In terms of expertise, the group was comprised mainly of neuromuscular specialists and neuropediatricians, and also included a small number of geneticists and physicians with expertise in the management of adults with neuromuscular diseases.

\section{Methods}

The expert meeting, as described above, was convened to discuss and debate issues relating to the diagnosis and management of DMD patients in the MENA region. A written survey was conducted prior to the meeting to identify current practices in individual countries regarding referral processes, access to technical facilities, multidisciplinary expertise and staffing profile, timeline for diagnosis, diagnostic procedures, patient's natural history and outcome measures (Supplementary Materials 2).

The aim of this exercise was to better understand the level of care provided to children with DMD with the goal of identifying what works and what does not work in the MENA region so as to improve the overall level of care in terms of maintenance of functionality and a better quality of life, and ultimately, increased longevity.

The chair of the meeting developed the questionnaire as a discussion guide. The questions addressed different aspects relating to the overall care of patients with DMD in relation to the topics listed below:

1. Patient pathway and referral process;

2. Access to 'centers of excellence' and medical staff;

3. Diagnostic tools and timelines;

4. Natural history of DMD in the MENA region;

5. Patient registries and data management;

6. Current treatment of DMD;

7. New treatment approaches;

8. Other management measures;

9. Obstacles in access to care for patients with DMD;

10. Patient education/awareness;

11. Expert recommendations and conclusions.

Each one of these topics was openly discussed with respect to global standards and regional (MENA) performance, and was guided by feedback and issues that arose from the survey.

\section{Discussion}

Patient pathway \& referral process

In the MENA region, there is not a standardized referral pathway for DMD patients and they are referred from a multitude of sources which varies from country to country. This contributes to delays in confirming the diagnosis and initiating management. Currently, the main sources of referral are from community neurologists, pediatricians (particularly when there are behavioral problems), family physicians and gastroenterologists (when patients present with incidental findings of elevated liver enzymes). Geneticists may also be involved in cases related to family 
history. Interestingly, family physicians were generally the first to refer the patients for a genetic diagnosis. Some children are brought directly to the clinic by their parents and/or relatives almost exclusively as a result of concerns regarding a family history.

The expert group discussed the benefits of early referral, which generally equates to early diagnosis, and can facilitate earlier management and improved clinical outcomes. This should therefore be an important objective moving forward. The major sign of DMD is muscle weakness, and this is the most common reason for physician consultation. It can present as delayed or difficulties in walking and an increased risk of falling. Elevated serum creatine kinase $(\mathrm{CK})$ levels are also a frequent reason for the referral of patients with DMD from both general practice and specialist care facilities.

Disease awareness campaigns, in particular pertaining to the signs and symptoms of the disease, should target both the medical community and the general public to help improve early diagnosis.

\section{Access to 'centers of excellence' \& medical staff}

The expert group indicated that another key unmet need in the management of DMD in the MENA region is the availability of - and proximity to - diagnostic and treatment centers. There is currently no standardized diagnostic process across the region, and clinical practice tends to differ between and within countries. The expert group noted that in Europe, doctors are generally well-trained to test CK levels in any male child who exhibits motor and/or developmental delays, and this does not occur consistently in the MENA region. Additionally, there are regional differences in that some countries have access to the genetic diagnostic testing, but others do not. If a person lives outside a metropolitan area, the chances of an early diagnosis are much lower. Genetic clinics are available in some hospitals, and in these institutions it should be easier for general physicians and pediatricians to access this expertise and detect DMD early. Currently, genetic testing is only partly available in the MENA region. In some instances, DNA extraction and multiplex ligation-dependent probe amplification (MLPA) testing might be performed locally, but in most cases, physicians and centers send samples to the USA or Europe for further assessments, including sequencing of the $D M D$ gene in search of specific mutations.

Because of the nature of the disease, DMD management requires a multidisciplinary approach involving different specialties (Figure 1). Mixed responses were received from the expert group regarding the availability of such specialties in their institutions/countries. Geneticists, pediatricians, neuromuscular experts and physiotherapists play a significant role in the early phase of DMD to establish the diagnosis and to help guide the initial therapeutic approach. Physiotherapists, pulmonologists and cardiologists play a more prominent role in the later phases of the disease when the disability and muscle dystrophy have spread to impact pulmonary and cardiac function. In the MENA region a multidisciplinary approach is only usually available in tertiary care centers, and in most instances, these specialties are in different clinics within the center. The communication and coordination required between these clinics for optimal patient follow-up and care is generally lacking. The expert group concluded that the development of referral and expert centers with centralized expertise and multidisciplinary services would be a large step in the right direction to improve DMD patient management.

\section{Diagnostic tools \& timelines}

The expert group unanimously agreed that to improve the treatment of patients with DMD, early and accurate diagnosis is essential. Overall, DMD awareness remains somewhat low in the MENA region and there is a knowledge gap both in the general public and the medical community. Currently, the average age for DMD diagnosis is 7-8 years for an affected first child and slightly earlier for a second child in the same family. The mean age at diagnosis has been reported to be 4.3 years in the UK with a delay in diagnosis averaging $2-3$ years [6]. As noted by these authors, earlier diagnosis is likely to result in better opportunities for optimized care and should be the target [6].

The most common and frequently followed diagnostic procedures are serum CK testing, genetic testing, muscle biopsy and neurological assessments. The expert group recognized that there is no standard diagnostic algorithm currently available in the MENA region (e.g., Figure 2). In relation to the available diagnostic tests, physicians prefer to use the MLPA test since it simultaneously evaluates all 79 exons on the DMD gene and identifies the copy number variation (deletion or duplication) in approximately $70 \%$ of DMD patients [7]. When the MLPA test fails to identify the genetic mutation, sequencing of the $D M D$ gene may be required to provide a genetic diagnosis in the remaining $30 \%$ of patients with other mutation types such as point mutations [7].

MLPA testing to check for DMD is the most common diagnostic procedure, although not every center can perform this test and samples are often sent abroad to be analyzed. Gene sequencing samples are usually sent to the 


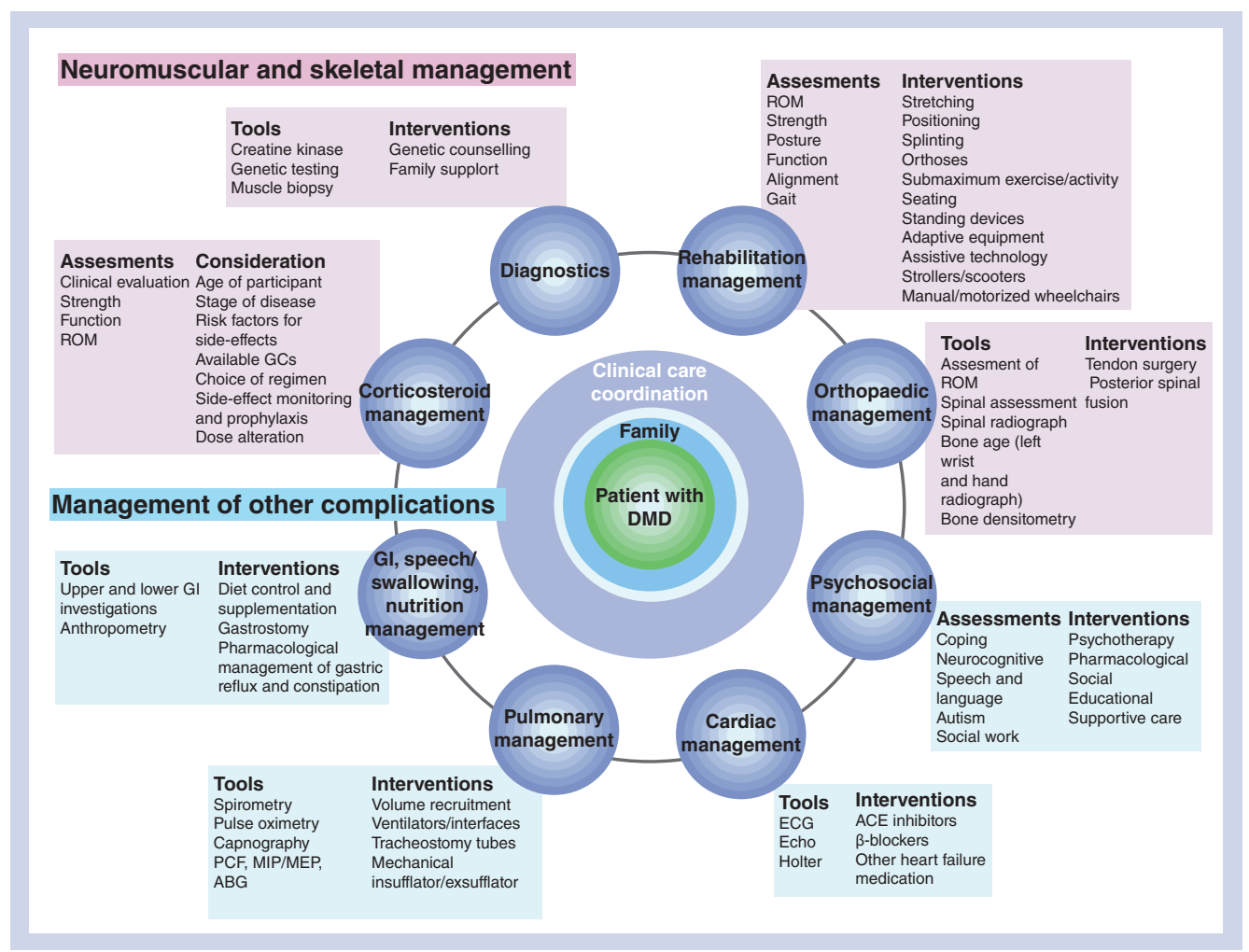

Figure 1. Multidisciplinary approach to Duchenne muscular dystrophy management.

DMD: Duchenne muscular dystrophy.

Reproduced from [1] with permission of Elsevier Ltd.

USA or Europe and this further delays diagnosis due to processing and shipping times. In some cases, patients are referred to a neurologist who will often request a CK test. If a child has CK levels above $5000 \mathrm{U} / 1$, genetic testing is then performed.

Based on the laboratories that are available, there can be a significant cost difference for dystrophin gene sequencing versus MLPA testing in some countries. The expert group discussed the affordability of diagnostic testing and for the majority it was a major concern, particularly if the samples needed to be sent abroad. The availability of a regional center for dystrophin gene sequencing was a topic that all participants agreed would be of benefit in the region, both in terms of cost and more rapid diagnosis. However, there may be local issues to overcome since some institutions may be bound by contractual arrangements between their hospital laboratory and the referral laboratory.

The lack of a regional gene sequencing program/laboratory, the logistical obstacles involved in sending blood (DNA) samples abroad, the costs involved in this exercise and the long turnaround times are all factors that currently contribute to potential delays in diagnosis and treatment for the patient, and genetic counseling for the families.

\section{Natural history of DMD in the MENA region}

Although epidemiology data for DMD in the MENA region is lacking, it was the view of the expert group that based upon their experience in clinical practice, the natural history was similar to what has been observed globally. In the last 20-30 years, treatment of patients with DMD - particularly better respiratory care - has improved longevity such that some patients are now surviving into their 30s or 40s [8]. The majority of individuals remain ambulatory up to the age of 8-12 years, but in their teenage years an increasing number become wheelchair bound.

Dystrophin production is affected from birth but, as a result of compensatory mechanisms, symptoms of DMD (usually motor dysfunction) do not generally appear until the age of 3 years [6]. Initially, prior to diagnosis, children usually present with delayed walking or delayed speech compared with their peers. In the late ambulatory stage, motor symptoms deteriorate and walking becomes increasingly difficult. The child experiences problems such as difficulty in getting up from the floor and climbing stairs, and frequently falls [9]. 


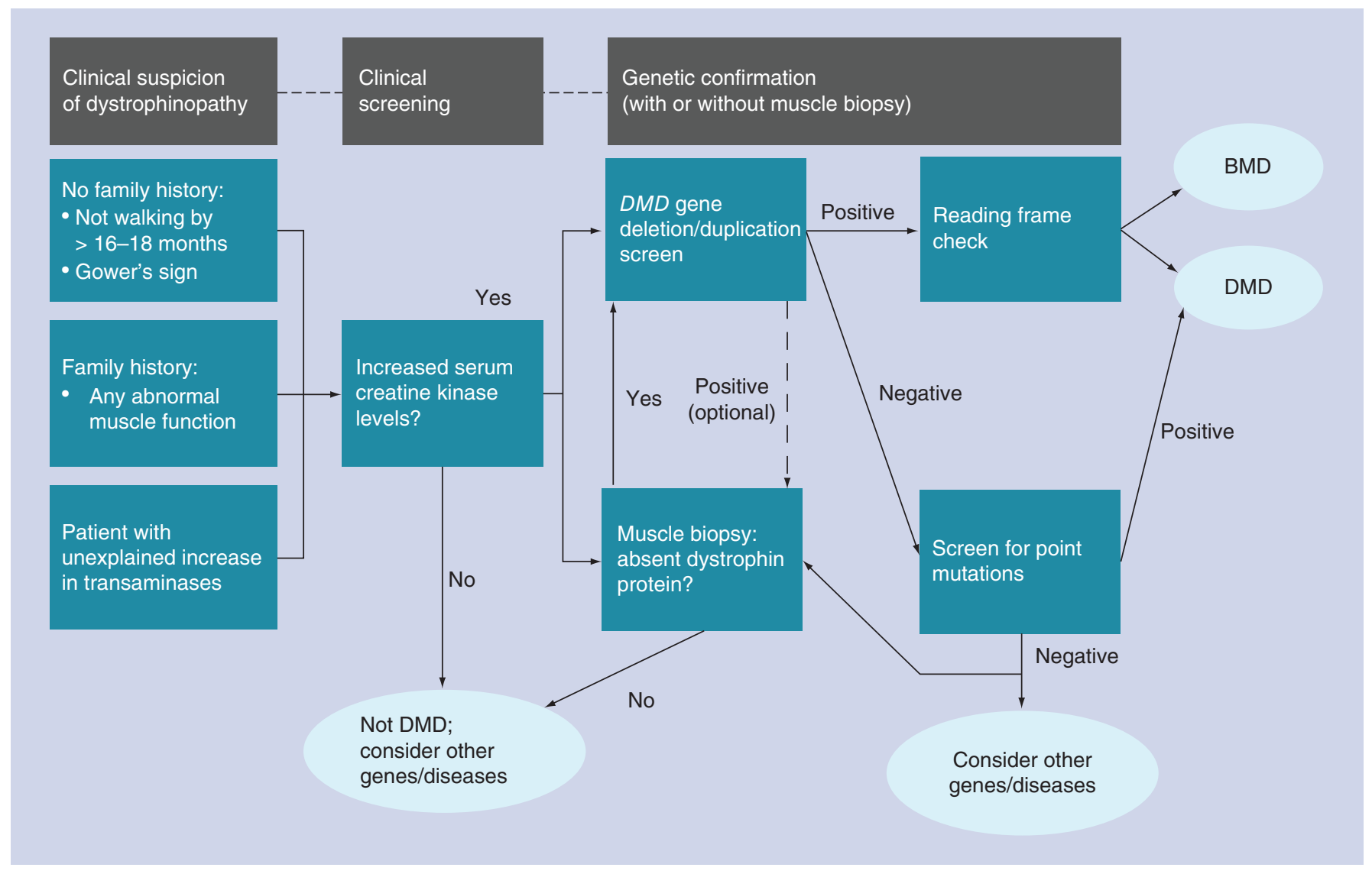

Figure 2. Diagnostic pathway for Duchenne muscular dystrophy patients. DMD: Duchenne muscular dystrophy.

Adapted from [1].

With time, the signs of DMD become more noticeable and the four classical DMD motor signs are:

- Gowers' maneuver: patients need to support themselves with hands on thighs when they get up from the floor;

- Waddling gait (myopathic gait);

- Toe-walking;

- Stair climbing: patients bring the second foot up to join the first rather than going foot over foot, in other words, climbing each stair one step at a time.

Natural history data show that up to the age of 7 years, 6-min walking test (6MWT) results are generally stable or improve [10]. This is followed by a period of stability, particularly in children with higher levels of baseline function (6MWD $>350 \mathrm{~m})$. However, at a certain stage, children enter a more rapid decline phase where they experience a substantial decrease in walking ability over a period of 1 year. Evolving natural history data show that boys are at high risk for precipitous losses in ambulation when a clinical threshold value of muscle loss occurs; this typically corresponds with a 6MWT of approximately $\leq 300 \mathrm{~m}$ (Figure 3). While muscle weakness and subsequent loss of function is inevitable, disease progression and the rate of decline are heterogeneous and a wide variability of disease progression between patients has been recorded [11].

Boys with DMD have decreased bone density and increased risk of fractures and 35-40\% of them result in permanent loss of ambulation [12,13]. Loss of mobility and consequent wheelchair dependence usually occurs between the ages of 9 and 15 years [14-16] and a general weakening of the back muscles results in the development of scoliosis. Treatment with corticosteroids helps improve posture and arm strength and reduces the need for surgery for scoliosis, and there is clear evidence for longer term benefit ( 2 years or longer), including improved respiratory 


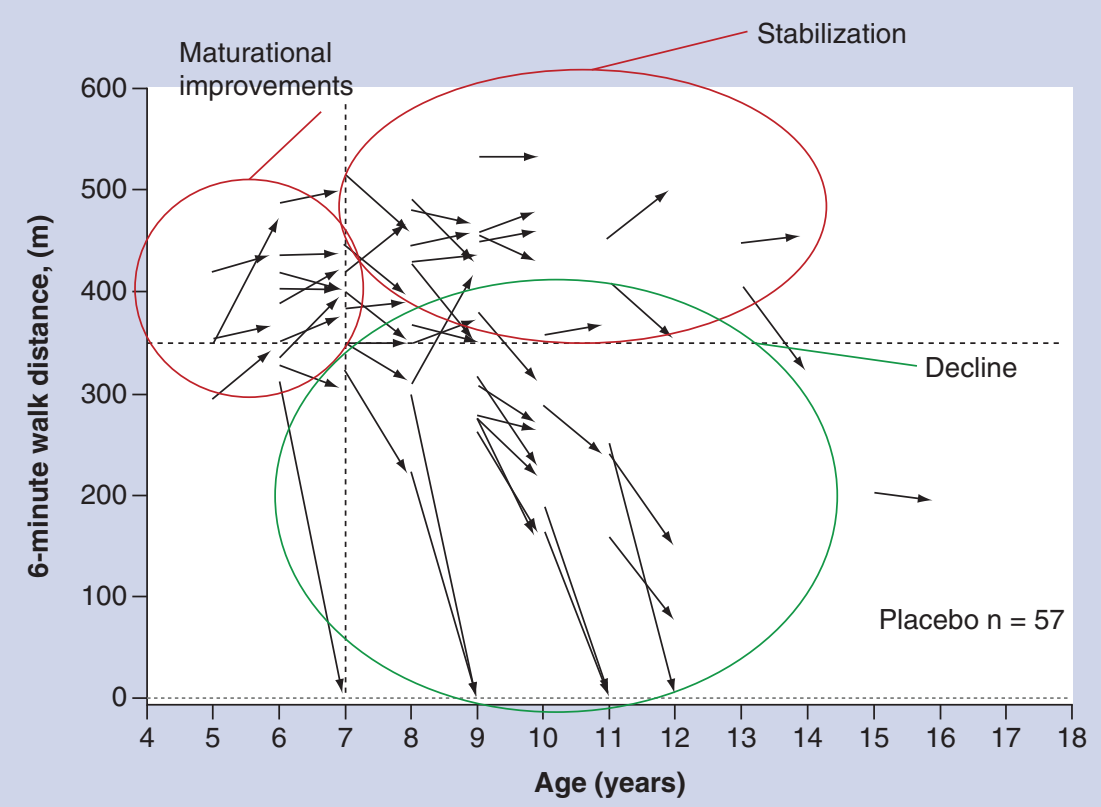

Figure 3. The natural history of Duchenne muscular dystrophy as defined by change in 6-min walking test from baseline to 48 weeks from the placebo group in study 007. Presented by Spiegel et al. at the 41 Annual Meeting of the Society for Neuropediatrics, Basel, Switzerland, 23-26 April 2015.

and possibly cardiac function $[17,18]$. Nevertheless, patients eventually develop respiratory symptoms and they are at a high risk of both respiratory and cardiac deterioration [8].

In the later stage of the disease, complications become more severe and continued deterioration of muscle strength and permanent use of a wheelchair usually leads to respiratory insufficiency and need for ventilation support; initially at night, but eventually at all times. Dependence on permanent ventilation, which may require tracheostomy, usually occurs before the patient reaches their mid-20s [19,20]. Cardiac complications also occur, especially as the disease progresses, with cardiomyopathy diagnosed in over $50 \%$ of adults with DMD [21].

Improvements in care in recent years have seen some benefits in longevity, but most patients with DMD still die from heart or lung failure at a relatively early age with only a few patients surviving beyond their third decade of life [22]. Several European studies provide survival estimates for patients based on the care provided. The average age of death in studies published between 2007 and 2013 was between 20 and 25 years (overall range 10-46 years in a Swedish cohort) [20,22-25] with improved longevity recorded in patients undergoing spinal surgery and receiving ventilation [25]. As noted earlier, the benefits of improved patient management, particularly providing ventilator support for lung function, has resulted in some patients surviving into the fourth decade of life [7,20,22,24].

\section{Patient registries \& data management}

A major obstacle in understanding DMD in the MENA region, its prevalence, incidence and the distribution of the different mutations, is the lack of methodologically sound and large-scale epidemiological studies. The majority of participants at the meeting confirmed that they keep personal databases on their patients, but there is no national or regional demographic information.

The expert group unanimously agreed that a patient registry would be a valuable initiative to identify epidemiological trends and a useful starting point for initiating small studies. There are many questions that will need to be answered if a registry is to be set up: which neuromuscular diseases should be included; what meta-data should be recorded; standards for entries (to avoid duplication); funding and rules for updating (important for diseases such as DMD where the patient's condition can change rapidly)? As the starting point, the group discussed the possibility of using/working with the Treat-NMD registry (www.treat-nmd.eu). 
Table 1. Experts' recommendations \& conclusion.

\begin{tabular}{|c|c|}
\hline Subject & Recommendations \\
\hline Patient pathway and referral process & $\begin{array}{l}\text { Education and a strong focus on increasing the recognition of early symptoms could save valuable time and improve } \\
\text { disease prognosis for the patients referred to specialized centers that have the multidisciplinary services needed to } \\
\text { manage DMD } \\
\text { Disease awareness is important for specific specialties such as pediatrics, large GP centers and } \\
\text { physiotherapy/rehabilitation centers where children are receiving treatment; and also in the general population } \\
\text { General disease awareness campaigns and scientific gatherings are needed to recognize signs and symptoms of DMD } \\
\text { as early as possible to ensure that affected children are referred to pediatric neuromuscular experts where diagnosis } \\
\text { and a holistic approach to therapy can be provided }\end{array}$ \\
\hline $\begin{array}{l}\text { Access to 'centers of excellence' and } \\
\text { medical staff }\end{array}$ & $\begin{array}{l}\text { Referral centers need to be developed and staffed with the required expertise and provided with optimal technical } \\
\text { facilities. In some instances, patients living in remote areas and outside of the large urban centers do not get the } \\
\text { right medical care } \\
\text { The ability to provide the required multidisciplinary approach is of paramount importance. One way of educating } \\
\text { stakeholders is through different courses that would include both theoretical and practical learning }\end{array}$ \\
\hline Diagnostic tools and timelines & $\begin{array}{l}\text { Improving the awareness of general pediatricians and family medicine doctors of the key diagnostic features of DMD } \\
\text { is crucial for the early diagnosis of DMD and will be a big step forward } \\
\text { Gene sequencing in patients that have received a negative MLPA test result, and in those that would like to send } \\
\text { their samples for analysis in the first instance prior to performing an MLPA test, is currently supported and needs to } \\
\text { be maintained. There is great value in this service since it facilitates earlier diagnosis and reduces cost and turnaround } \\
\text { times } \\
\text { Genetic diagnostics should be locally available. There are several regional labs that are acquiring the technology and } \\
\text { will soon be able to perform full gene sequencing and diagnosis for DMD patients. This should reduce logistical } \\
\text { hurdles, costs and the time for diagnosis } \\
\text { It would be helpful to develop a regional (MENA) approach to DMD management: for example, agree a diagnostic } \\
\text { algorithm for the region and share more of the best practices to support a harmonized approach }\end{array}$ \\
\hline Patient registries and data management & $\begin{array}{l}\text { The region lacks consolidated epidemiological data at all levels (i.e., regional or by country); each center keeps its } \\
\text { own records without coordination } \\
\text { Patient registries are needed to gather information on patient populations, understand the genetic variations in the } \\
\text { region, and to support research and scientific publications } \\
\text { Saudi Arabia has initiated a national registry for neuromuscular diseases and this should be replicated in other } \\
\text { countries and coordinated regionally }\end{array}$ \\
\hline $\begin{array}{l}\text { Current management and treatment } \\
\text { follow up for DMD }\end{array}$ & $\begin{array}{l}\text { A multidisciplinary approach will provide the highest standards of patient care. This can be achieved by setting up } \\
\text { 'centers of excellence' to follow DMD patients and provide full service including respiratory, cardiac and } \\
\text { physiotherapy expertise } \\
\text { Rehabilitation and physiotherapy are an important part of follow-up care to maintain functional status and the } \\
\text { patient's quality of life }\end{array}$ \\
\hline Patient education/awareness & $\begin{array}{l}\text { There are isolated patient support groups in the MENA region, but the role of patient advocacy is limited } \\
\text { Provision of on-going education through different channels such as local language family guides on how to deal with } \\
\text { the disease and online portals in Arabic will empower patients and their families. This should help to reduce the } \\
\text { social stigma associated with diseases such as DMD } \\
\text { Families and family physicians have an important role to play in identifying the early signs and symptoms of DMD, } \\
\text { and referral of patients for further diagnosis. }\end{array}$ \\
\hline
\end{tabular}

\section{Current treatment of DMD}

Currently, corticosteroids are considered to be the gold standard of DMD treatment since they have been shown to slow progression of the disease and extend functional ability for 2 years or more [18]. However, the exact mechanisms by which they promote these benefits are poorly understood [17]. In the MENA region, about $50-80 \%$ of patients are maintained on corticosteroids such as prednisone/prednisolone and deflazacort, but different regimens are used and tolerability may be an issue [7]. The latter is important since it can have an impact on the adherence to treatment, which is essential to ensure a response to steroid therapy. Education of patients/parents to improve awareness of issues relating to corticosteroids such as expected benefits, possible side effects, compliance and diet is seen as a key initiative by the expert group. Available supportive therapy aims to alleviate disease symptoms and slow the rate of disease progression; however, it does not address the underlying cause of the disease or prevent its ultimate course to more severe DMD with loss of walking ability and wheelchair dependence $[11,16]$. In addition, the benefits of corticosteroids must be balanced against a side-effect profile that presents significant challenges including excessive weight gain, increased risk of bone fractures, behavioral abnormalities, hypertension, cushingoid appearance and excessive hair growth. Not all boys can tolerate steroid therapy and this is problematic since this leaves them with no effective treatment and a poor prognosis. 


\section{New treatment approaches}

The last decade has seen growing interest in novel therapeutic approaches including genetic techniques (promotion of stop codon read-through and exon skipping, and gene therapy), cell therapy, myostatin inhibition, phosphodiesterase inhibition, ILGF1 modulation and utrophin upregulation being some of those described in recent years $[7,18]$. Many of these procedures are in the early stages of development and the two closest to use in everyday clinical practice are stop codon read-through and exon skipping.

Stop codon read-through

Approximately $15 \%$ of DMD patients have a nonsense mutation in the dystrophin gene [26] and ataluren has recently been developed to promote translational read-through of premature stop codons [27]. Ataluren is licensed as Translarna ${ }^{\circledR}$ within European Union (EU) Member States, Iceland, Liechtenstein, Norway, Israel and South Korea. In EU countries it is indicated for the treatment of DMD resulting from a nonsense mutation in the dystrophin gene, in ambulatory patients aged 2 years and older. Efficacy has not been demonstrated in patients who are no longer walking. Patients who do not have a nonsense mutation should not receive ataluren.

The expert group reported a number of different practices including initiating treatment with ataluren, alone or in combination with corticosteroids, and ataluren followed by dietary management and appropriate cardiac monitoring. At the present time, approximately half of the panel participants were using corticosteroids and the other half were using a combination of corticosteroids and ataluren. The overall expectation was that ataluren would slow disease progression depending on the stage of the disease. They also noted that clinical trials with ataluren were generally in patients being treated with corticosteroids, and they recommended that patients with nonsense mutation DMD should be treated with corticosteroids for 6 months before ataluren is started.

\section{Exon skipping}

Eteplirsen (Exondys $51^{\circledR}$ ) is an antisense oligonucleotide that is designed to bind to exon 51 of dystrophin premRNA, resulting in exclusion of this exon during mRNA processing in patients with genetic mutations that are amenable to exon 51 skipping. Exon skipping is intended to allow for production of an internally truncated dystrophin protein. By increasing the quantity of an abnormal, but potentially functional, dystrophin protein, the objective is to slow or prevent the progression of DMD [28]. Eteplirsen has been evaluated in clinical trials involving small numbers of patients and has been approved by the US FDA under an accelerated approval based on an increase in dystrophin in skeletal muscle observed in some patients.

\section{Other management measures}

Dietary advice (sometimes gastric feeding), preventing and treating bone-related problems and managing complications of long-term corticosteroid therapy may be required, as well as psychosocial support. Input from different specialties with emphasis on therapeutic issues will change as DMD progresses, for example, dietary and orthopedic advice [1]. Regarding treatment follow-up, in the absence of specific guidelines, the participants in the expert group provided a variety of responses. Overall, they agreed that, depending on the individual, monitoring of muscle function/motor performance or CK testing should be performed, along with physiotherapy and rehabilitation for more advanced cases.

Progressive scoliosis develops in the majority of DMD patients as a combined result of wheelchair dependence, paralysis of the extensor muscles, contractures and growth spurts [29]. Physical therapy and the use of splints/orthoses can be useful in some patients and surgical intervention may be required for lower-limb contractures. In later childhood, inpatient spinal surgery, occupational therapy and rehabilitation may be required to correct scoliosis and there is also a greater need for inpatient orthopedic, cardiac and respiratory intervention, including assisted ventilation.

Due to the lack of proper rehabilitation and the absence of multidisciplinary follow-up in many instances, surgery and/or invasive procedures are not preferred options for nonambulatory patients. Another important consideration is the use of noninvasive ventilators to deal with nocturnal hypoventilation, sleep-related breathing difficulties and ultimately respiratory failure.

The above discussions highlight the need for a multidisciplinary care approach to the management of patients with DMD. The expert group recommended the setting up of 'centers of excellence' to follow DMD patients long-term to provide them with the necessary care (cardiovascular, neuromuscular, occupational, physiotherapy and respiratory) to maintain the best achievable functional status and quality of life. 


\section{Obstacles in access to care for patients with DMD}

In this era of personalized medicine and as new mutation-specific therapies emerge, there is now an increasing hope for patients (and their families) with some genetic disorders. Nevertheless, several obstacles exist in the MENA region that prevents access of medicines such as ataluren and eteplirsen to all eligible patients, including:

\section{1) Reimbursement \& cost of the medication:}

Governments of the region provide healthcare coverage for nationals while expatriates are dependent on their private insurance schemes that usually do not cover rare diseases or pre-existing conditions. When therapy can be provided in public hospitals, the cost of the treatment remains an issue. Dosage is weight-based and therapy is long-term, hence increasing the costs on a year-by-year basis.

2) Appropriate monitoring \& follow-up of these patients:

In the absence of effective treatments, at least up until very recently, patients and their families often became despondent and lost hope. In such circumstances diagnosed patients tended to stop attending follow-up visits. Education, increasing awareness, incorporation of an appropriate multidisciplinary follow-up schedule and setting realistic treatment objectives are keys to managing DMD patients and keeping them in the healthcare system.

3) Obstacles at the national level:

Many patients with DMD go undiagnosed due to a lack of awareness and education among the general public (including parents), as well as the absence of appropriate large-scale diagnostic screening programs in the MENA region.

\section{Patient education/awareness}

Given the challenges of raising a child with DMD and the poor long-term prognosis, genetic consultation before marriage is important if there is a family history. Some governments support counseling for young couples; but there is no mechanism for enforcing the recommendations provided by geneticists to couples planning to marry. If pre-implantation genetic diagnosis is possible then this would be ethically and religiously more acceptable; however, it is not widely available and is very expensive and is associated with high failure rates.

There are several cultural and social factors in the region that reinforce the stigma associated with genetic diseases such as DMD. Educating the extended family may help in addressing the problem at home and in society at large. However, genetic counseling remains a challenge in the MENA region.

Table 1 summarises the main recommendations and conclusions made by the Experts Group.

\section{Summary points}

- Duchenne muscular dystrophy (DMD) is a severe and rare X-linked neuromuscular childhood disorder that results in functional decline, loss of ambulation and early death due to cardiac or respiratory failure.

- This White paper reviews the current management of DMD in the Middle East, North Africa (MENA) region based upon local expert opinions and recommendations.

- Ideally, DMD management should involve a multidisciplinary approach.

- However, few tertiary care hospitals in the region are currently able to provide the full spectrum of medical expertise and services needed by DMD patients, and clinical practice in the region remains heterogeneous.

- Specific guidelines for diagnosis and treatment are needed in the MENA region to improve outcomes.

- Disease awareness among the general public and the medical community is generally poor and needs to be improved.

- The development and wider study of mutation-specific therapies necessitates improvement and more general distribution of education programs regarding the disease (e.g., to help recognize early signs and symptoms).

- Furthermore, standardized referral and diagnosis pathways, patient registries and support groups will significantly improve the management of the disease. 
Author contributions

All authors contributed equally to the survey and scientific meeting in Dubai (and subsequent discussions) upon which this report is based. All authors approved the final decision to submit the article for publication.

Financial \& competing interests disclosure

This work was supported by financial grants from PTC Therapeutics and Genpharm services. The authors have no other relevant affiliations or financial involvement with any organization or entity with a financial interest in or financial conflict with the subject matter or materials discussed in the manuscript apart from those disclosed.

Editorial assistance on behalf of the authors was provided by S Clissold of Content Ed Net and was funded by PTC Therapeutics and Genpharm services.

\section{Open access}

This work is licensed under the Attribution-NonCommercial-NoDerivatives 4.0 Unported License. To view a copy of this license, visit http://creativecommons.org/licenses/by-nc-nd/4.0/

\section{References}

1. Bushby K, Finkel R, Birnkrant DJ et al. Diagnosis and management of Duchenne muscular dystrophy, part 1: diagnosis, and pharmacological and psychosocial management. Lancet Neurol. 9, 77-93 (2010).

2. Snow WM, Anderson JE, Jakobson LS. Neuropsychological and neurobehavioral functioning in Duchenne muscular dystrophy: a review. Neurosci. Biobehav. Rev. 37, 743-752 (2013).

3. Taylor PJ, Betts GA, Maroulis S et al. Dystrophin gene mutation location and the risk of cognitive impairment in Duchenne muscular dystrophy. PLoS ONE 5(1), e8803 (2010).

4. Kohler M, Clarenbach CF, Böni L, Brack T, Russi EW, Bloch KE. Quality of life, physical disability, and respiratory impairment in Duchenne muscular dystrophy. Am. J. Respir. Crit. Care Med. 172, 1032-1036 (2005).

5. Birnkrant DJ, Yilmaz O, Nicolai T, Black JB, Mhanna MJ, Noah TL. Pediatric pulmonology year in review 2015: Part 3. Pediatr. Pulmonol. 51, 747-753 (2016).

6. van Ruiten HJ, Straub V, Bushby K, Guglieri M. Improving recognition of Duchenne muscular dystrophy: a retrospective case note review. Arch. Dis. Child 99, 1074-1077 (2014).

7. Falzarano MS, Scotton C, Passarelli C, Ferlini A. Duchenne muscular dystrophy: from diagnosis to therapy. Molecules 20(10), 18168-18184 (2015).

8. LoMauro A, D’Angelo MG, Aliverti A. Assessment and management of respiratory function in patients with Duchenne muscular dystrophy: current and emerging options. Ther. Clin. Risk Manag. 11, 1475-1488 (2015).

9. McDonald CM, Henricson EK, Han JJ et al. The 6-minute walk test as a new outcome measure in Duchenne muscular dystrophy. Muscle Nerve 41, 500-510 (2010).

10. McDonald CM, Henricson EK, Abresch RT et al. The 6-minute walk test and other endpoints in Duchenne muscular dystrophy: longitudinal natural history observations over 48 weeks from a multicenter study. Muscle Nerve 48, 343-356 (2013).

11. Goemans N, Kirschner J, Mercuri E. New perspectives in the management of Duchenne muscular dystrophy. Eur. Neurological Rev. 9, 78-82 (2013).

12. McDonald CM. Physical activity health impairments, and disability in neuromuscular disease. Am. J. Phys. Med. Rehabil. 81(11 Suppl.), S108-S120 (2002).

13. Vestergaard P, Glerup H, Steffensen BF, Rejnmark L, Rahbek J, Moseklide L. Fracture risk in patients with muscular dystrophy and spinal muscular atrophy. J. Rehabil. Med. 33, 150-155 (2001).

14. Humbertclaude V, Hamroun D, Bezzou K et al. Motor and respiratory heterogeneity in Duchenne patients:implication for clinical trials. Eur. J. Paediatr. Neurol. 16, 149-160 (2012).

15. Moxley RT 3rd, Pandya S, Ciafaloni E, Fox DJ, Campbell K. Change in natural history of Duchenne muscular dystrophy with long-term corticosteroid treatment: implications for management. J. Child Neurol. 25, 1116-1129 (2010).

16. Ricotti V, Ridout $\mathrm{Da}$, Scott $\mathrm{E}$ et al. Long-term benefits and adverse effects of intermittent versus daily glucocorticoids in boys with Duchenne muscular dystrophy. J. Neurol. Neurosurg. Psych. 84, 698-705 (2013).

17. Griggs RC, Herr BE, Reha A et al. Corticosteroids in Duchenne muscular dystrophy: major variations in practice. Muscle Nerve 48, 27-31 (2013).

18. Scully MA, Cwik VA, Marshall BC, Ciafaloni E, Wolff JM, Getchius TS, Griggs RC. Can outcomes in Duchenne muscular dystrophy be improved by public reporting of data? Neurology 80, 583-589 (2013).

19. Ishikawa Y, Miura T, Ishikawa Y, Aoyagi T, Ogata H, Hamada S, Minami R. Duchenne muscular dystrophy: survival by cardio-respiratory interventions. Neuromuscul. Disord. 21, 47-51 (2011). 
20. Kieny P, Chollet S, Delalande P, Le Fort M, Magot A, Pereon Y, Perrouin Verbe B. Evolution of life expectancy of patients with Duchenne muscular dystrophy at AFM Yolaine de Kepper centre between 1981 and 2011. Ann. Phys. Rehabil. Med. 56, 443-454 (2013).

21. Rodger S, Woods KL, Bladen CL et al. Adult care for Duchenne muscular dystrophy in the UK. J. Neurol. 262, 629-641 (2015).

22. Passamano L, Taglia A, Palladino A et al. Improvement of survival in Duchenne Muscular Dystrophy: retrospective analysis of 835 patients. Acta Myol. 31, 121-125 (2012).

23. Stromberg A, Darin N, Kroksmark A. What was the age and cause of death in patients with Duchenne muscular dystrophy in Sweden during 2000-2010? Neuromusc. Disord. 22, 880-881 (2012).

24. Rall S, Grimm T. Survival in Duchenne muscular dystrophy. Acta Myol. 31, 117-120 (2012).

25. Eagle M, Bourke J, Bullock R et al. Managing Duchenne muscular dystrophy - the additive effect of spinal surgery and home nocturnal ventilation in improving survival. Neuromusc. Disord. 17, 470-475 (2007).

26. Finkel RS. Read-through strategies for suppression of nonsense mutations in Duchenne/Becker muscular dystrophy: aminoglycosides and ataluren (PTC124). J. Child. Neurol. 25, 1158-1164 (2010).

27. Haas M, Vlcek V, Balabanov P et al. European Medicines Agency review of ataluren for the treatment of ambulant patients aged 5 years and older with Duchenne muscular dystrophy resulting from a nonsense mutation in the dystrophin gene. Neuromusc. Disord. 25, 5-13 (2015).

28. Mendell JR, Lloyd-Puryear M. Report of MDA muscle disease symposium on newborn screening for Duchenne muscular dystrophy. Muscle Nerve 48, 21-26 (2013).

29. Sanzarello I, Merlini L, Traina F, Rosa MA, Faldini C. Corticosteroid treatment impact on spinal deformity in Duchenne muscular dystrophy. Int. Sch. Res. Notices 2014, 965235 (2014). 
White Paper Jumah, Muhaizea, Rumayyan et al. 